\title{
Research on the Development of Biomedical Engineering and Modern Medical Equipment
}

\author{
Yang Bin, Wang Guangchang
}

School of Humanities and Information Management, Chengdu Medical College, Chengdu, China

Keywords: Biomedicine; Engineering; Medical Treatment; Equipment; Equipment

\begin{abstract}
This paper mainly discusses the concept, development status and research scope of biomedical engineering and its application in medicine. This paper analyzes the main classification of modern medical equipment and equipment from functional aspects, and advances the development direction of biomedical engineering and modern medical equipment.
\end{abstract}

\section{Introduction}

The two major engineering disciplines of life sciences, including biotechnology and biomedical engineering, have developed rapidly and intersect with almost all areas of nature and engineering. BioMedical engineering (BioMedical Engineemg, BME) is the application of engineering theory and method, on the prevention and treatment of diseases in the medical field, safeguard human health of the edge of a new science, is the modern life science and medicine, engineering, and the combination of development, its formation and development of model basically can be summarized as: through engineering technology in the science of physics, chemistry and technology and new technology, principle, method applied to the development of medical devices, equipment, materials, meet the needs of the clinical diagnosis and treatment. Along with the progress of science and technology, new methods of physics, chemistry, and engineering technology have been applied to medicine, more and more medical products, the corresponding industry more and more developed, has developed into on behalf of a country by the end of the twentieth century scientific and technological progress and the level of modernization symbol of the national economy. In the 20th century, BME technology has made great achievements, medical imaging diagnosis, clinical laboratory testing, functional imaging diagnostic technique progress, a variety of supported by new medical treatment equipment new treatments are emerging, represented by artificial organs such as heart pacemakers has gained popularity and development of artificial organs or parts on the basis of the new materials of adjuvant therapy equipment widely used, many new technology and new equipment across age emerge in endlessly. At present, the main branch of biomedical engineering field are: biomechanical and biological rheology, biological materials and artificial organs, the modeling and control of biological systems, biomedical information detection and processing, medical imaging and image processing, medical materials and nano technology, etc. Although the breadth of biomedical engineering, large amount of information, the development is rapid, but, according to its final research goal, which can be divided into biomedical materials, biomedical engineering equipment, remote diagnosis system and biological medical rehabilitation engineering. To this end, the technical personnel engaged in medical work must obtain the latest information through various channels, and constantly update the knowledge to adapt to the rapid development of modern science and technology.

\section{Application scope of medical equipment and equipment.}

The medical equipment and equipment includes diagnostic equipment, treatment equipment and auxiliary materials application, etc. The SFDA has a detailed classification. functionally

There are several main categories.

(1) medical imaging: X-ray photography and fluoroscopy, CT, MR, DSA, CR, DR, US imaging, ECT, PET. 
(2) clinical inspection, analysis, and molecular biology: the blood cell analyzer, biochemical analyzer, elisa analyzer, flow cytometry, blood coagulation analyzer instrument, fluorescence analyzer, free analyzer, PCR, DNA analyzer.

(3) the electrophysiological information detection and monitoring: ecg, eeg, emg, blood flow, blood sugar, $\mathrm{pH}$, guide more physiological monitor (ecg, heart rate, blood pressure, blood oxygen, impedance breathing and body temperature), 24 hour dynamic monitor (ecg, eeg blood pressure), the operation monitoring equipment, maternal and infant monitor, fetal monitor, monitor of sleep.

(4) physical therapy, radiotherapy and stereotactic radiosurgery surgery (7 dao, x knife, particles), ultrasonic treatment technology (high strength focused ultrasound and ultrasound in vitro rubble, ultrasonic bleeding), the electromagnetic heat technology (in vitro warm treatment, body cavity warm physical therapy and heat setting) high energy tumor hyperthermia technology (high strength polyethylene ultrasound hyperthermia, rf heat therapy machine, microwave thermal therapy).

(5) minimally invasive: endoscope, robot, navigation and positioning.

(6) artificial organ replacement: artificial kidney, artificial liver, extracorporeal circulation.

(7) scalpel: electric knife, ultrasonic knife, vessel ligation.

(8) biomedical materials: surgical sutures, artificial skin, concealed glasses, artificial blood vessels, artificial tendons, teeth, bone prostheses, implants, catheters, and scaffolds.

(9) medical information System: PACS(Picture Archiving and Communication System), DICOM3. 0 (Digital Imaging and Communication in Medicine, 3. 0), IHE(for the Heahhcare Enterprise).

\section{Biomedical engineering and the development of modern medical equipment and equipment.}

In the future, medical devices will continue to develop in medical diagnosis, surgery, monitoring, rehabilitation and other aspects. The general trend is to be intelligent and simplified. Health to family; Cross integration in biology, physics and engineering design; To improve the accuracy of clinical diagnosis. The FDA experts predict the future ten years would be a big development on the following six aspects: including computer aided diagnosis, intelligent devices, biological sensors, robot, network, computer related technology; Molecular medicine; Family and self-care; Minimally invasive method; Equipment/drug combination products; Artificial organ transplantation/auxiliary device and tissue engineering components. The specific content is:

(1) genetic diagnosis: diagnostic instruments for genetic conditions and acquired genetic variation, such as DNA and nucleic acid analyzer.

(2) genetic treatment: a genetic treatment device for genetic conditions and genetic variation, such as a cell separator that purifies malignant bone marrow cells.

(3) tissue engineering apparatus: instruments made of biological materials. For example, biological replacement heart valve, artificial skin.

(4) computer-aided diagnosis: products that can optimize the treatment and improve the diagnostic level of the instrument, such as the "smart" patient card.

(5) smart devices: intelligent internal devices can automatically adjust the device's performance after input, such as implantable cardiac pacemaker.

(6) network of devices: the interconnection system of single devices enables it to have the performance of comprehensive system level, such as multiple anesthesia system.

(7) robots: robotic devices and instruments used for diagnosis and treatment can be more accurate and invasive than conventional operations under the control of surgeons.

Less injury.

(8) family/self-monitoring and diagnosis: non-clinical diagnostic instruments such as blood pressure monitor and pregnancy laboratory.

(9) family self-treatment: non-clinical setting of therapeutic devices, such as rehabilitation apparatus.

(10) electrical stimulation: an instrument that can affect tissue function, such as a pacemaker, with electromagnetic energy. 
(11) artificial organs: the replacement and replacement of hardware devices such as bone prostheses and heart valves.

(12) non-implantable auxiliary sensing: an external device, such as a hearing aid, that ACTS as an auxiliary function for the sensing function.

(13) telemedicine: device systems that can be diagnosed and treated at a distance, such as remote radiology and remote psychiatry.

(14) virtual technology: to provide object structure simulation devices for clinical applications, such as virtual colonoscopy.

(15) biosensor: used for diagnostic and monitoring devices with advanced or isolated sensors, such as oximeter, for DNA analysis.

Biological molecular integrated circuit chip.

(16) instruments, drugs and biological products: the application of medical devices and pharmacology and biological techniques to a compound instrument in a device, such as.

Perfusion pump, orthopedic prosthesis using bone growth factor.

(17) infection control instruments: instruments used to control the spread of infectious diseases, such as sterilizers.

(18) laser diagnosis and treatment: laser instruments for optical diagnosis and treatment of diseases, such as eye surgery lasers.

(19) medical imaging: non-invasive visual instruments for internal organs and functions, such as MRI, US, CT, ECT, DSA.

(20) micro devices: instruments based on tiny structures. For example, equipment manufactured by nanotechnology belongs to NEMS category.

(21) minimally invasive and non-invasive devices: greatly reduce the diagnosis and treatment of patient trauma, such as endoscope, arthroscopy and laparoscopy.

(22) nanomedicine materials and technologies: contrast agents and cell markers.

\section{Discussion on modern management of medical equipment.}

With the progress of modern society and the rapid development of science and technology, biomedical engineering plays an increasingly important role in modern hospitals. The role of high-precision medical equipment in medical work is irreplaceable, which makes the diagnosis quality and therapeutic effect of disease enter a whole new period. In general hospital medical instruments and equipment of a complete variety; Equipment scale and advanced degree is also an important symbol of modern hospitals, and today's medical equipment have the highest form of integrating modern science and technology, its professional knowledge involves all disciplines, is the essence of multiple disciplines. The update and increase of hospital equipment, the advanced technology performance, the equipment maintenance and maintenance requirements are also higher and higher; The maintenance time is urgent and the workload is increased. In order to meet the needs of modern hospital development, the maintenance, maintenance, maintenance and management of medical equipment should be properly recognized and treated. How to give full play to the function of medical equipment and ensure the normal operation of various equipment and equipment is an important issue in the development of hospitals. It is also an important factor for the survival of the hospital; The quality and utilization of the equipment directly affect the development of the hospital and the economic and social benefits. Therefore, the management of hospital medical equipment must be standardized, institutionalized, scientific and modernized. This paper discusses how to strengthen the management of medical equipment from several aspects.

\subsection{Demonstration, purchase and acceptance management of equipment.}

The procurement of hospital medical equipment, equipment and equipment must be purchased according to the rules and regulations [1]. Procurement procedures: ten thousand yuan of the following medical equipment, instrument and apparatus, equipment department according to the needs of hospital development and use of departments now, good management practices and procurement plan, submitted to the approval of the competent department, after the implementation 
step by step: for large or valuable equipment procurement, after requests (using) department summary (management) low reviews, argument convene experts (management department, department) low decision-making (dean).

Application shall be made by the head of the department in charge of the department in charge of the development direction and objectives of the department and the current situation.

Review and demonstration this link shall be convened by the management department to conduct the collective demonstration, market research and analysis benefits by the director of the department, the equipment department, the director and the relevant personnel. For the selected device after the argument, the choose and buy equipment to choose excellent performance and price, when the choose and buy equipment to solicit the opinions of the use and maintenance and maintenance engineering department staff, combined with the actual, full consideration the factors; For example, the source of the maintenance technical parts and consumables, as well as the technical operation training and related technical data and after-sales service of the staff members shall be embodied and committed in the order contract.

Purchasing public bidding Select multiple vendors to bid by the government procurement center to participate in, commission for discipline inspection to supervise, lead to grasp JiaGeGuan, equipment locked good technology, to ensure new equipment price is reasonable, good after-sales service, technical performance is good, can meet the needs of using department [5].

After the purchase of the acceptance equipment, the equipment shall be checked and accepted, and the inspection and acceptance shall be conducted jointly with the department, the operator, the maintenance engineer, the warehouse keeper and the archivist; According to the order contracts, invoices, packing according to the single technical performance of equipment, the technical data and accessories, accessories such as random by acceptance, fill in equipment acceptance certificate and acceptance of the whole process of installation, commissioning, to facilitate future use and repair and maintenance. All equipment and instruments are equipped with prominent operating procedures and operating procedure CARDS. Used after handle the warehousing department collect equipment and related accessories, for the needs of the complicated operation procedures guiding equipment operation and maintenance of equipment, technical data can be related to the technical data of copy to use department store; Other technical data in equipment files, maintenance spare parts in parts library, so that maintenance purposes, for the use of the equipment consumables plan in time, as soon as possible contact, ensure future use timely supply.

\subsection{Technical training of equipment.}

The equipment purchase and installation shall be transferred to the department for work, and the operation procedures and procedures shall be strictly followed and the special person shall be used and managed. Technical training must be done before operation. The training can be provided by the manufacturer to the site or to the installation site for technical training, or to the first-class hospital with similar equipment; The examination is qualified to take the post operation to use.

\subsection{Classification and archiving management of equipment.}

The filing, establishment and management of the equipment is carried out by the equipment specialist, which has a large labor intensity, long sorting cycle, complex retrieval and difficulty in borrowing. With the popularization of modern network technology, the establishment of network file system can reduce human resources and facilitate access and management. In the network management system in each device technical data files, including the order contracts, approval documents, reports, invoice copy, registration CARDS (purchase time, warranty period, sales unit, factory maintenance contact address, telephone number, fax number, head), operating rules and technical data, installation, maintenance and repair records, equipment usage, cost benefit analysis. The metering archives include the instrument statistics, the measurement requirements and standards of various instruments, the measurement results and the measurement period, the failure and repair records of the measuring instruments and so on. The data of these aspects are classified and encoded, and the archival catalogue is established for inspection and registration. It is an important project of hospital management to use microcomputer network to manage more 
convenient, labor-saving, time-saving and archiving management.

\subsection{Computer network management of equipment.}

This work is accomplished by microcomputer and equipment database is established.

All whole hospital medical equipment, instruments for statistics, registration, set up a register and the equipment name, type, manufacturer, price, contacts, address, telephone number, fax number, address, E-mail, date of warranty, use fixed number of year, enable and listed all the materials and accessories; If it is the medical equipment and equipment that has been scrapped in the near future, it is also required to register for the convenience of inspection.

For the management of (warehouse, procurement, delivery), when the equipment and instruments are in place, the equipment shall be classified into the warehouse. Library should check all kinds of equipment, input computer, and set up fixed assets, low loss, sanitary materials, scientific research and experimental materials, make classification arrangement, do a good job of "three certificates" verify and record use date, strictly to good quality. When the warehouse is issued, the implementation of the advanced first out, the old and new principles. Warehouse management to partition management system according to equipment classification, notice ventilated, moistureproof, heat, dust, corrosion protection, keep clean and tidy, prevent damage and the place and anti aging treatment, due to the continuous development of society, medical equipment, device replacement fast, inventory varieties and article is less of it or need to check inventory and in a planned way purchase, computer management and convenient query, greatly improving the work efficiency, make management personnel can fast, accurate, timely understand hospital for various devices.

\subsection{Maintenance, maintenance and maintenance management of the equipment.}

Medical equipment maintenance work according to the requirements of the ministry of health issued the tertiary facility maintenance implementation [3] : the daily maintenance of equipment and the level of maintenance shall be kept by the equipment user responsible for the requirements of management personnel familiar with equipment performance, usage and maintenance requirements. A variety of different nature of the equipment for maintenance and time interval, but clean, dust, heat, moisture, shock, anti-corrosion, dredge refueling, fastening adjustment, without exception, and so on and so forth is must be prepared to check equipment, maintenance process must be registered. Equipment department as a hospital equipment management department should regularly to each department is supervision and inspection of equipment maintenance of to implement maintenance work, maintenance work can greatly reduce the failure rate of the equipment.

Maintenance engineers must regularly maintain and maintain the equipment in charge of the equipment, so as to improve the utilization rate and service life of the equipment. When the problem is solved in a timely manner, the problem cannot be solved immediately to the superior department, and the relevant sales unit or the manufacturer's engineering staff consulting and processing method; The equipment should be able to repair the equipment when the equipment fails, and repair the equipment as soon as possible, and save money for the hospital.

The management of equipment maintenance is an important work which ensures the normal operation of the equipment and is the most important part of the equipment management. Because modern medical equipment is a composite product of the cutting-edge technology of various disciplines, it involves a wide range of technologies. The maintenance work requirement is high, the technical content is strong. So, to base on the reality, make full use of existing resources, reasonable allocation and use of resources allocation of maintenance personnel, implements the specialized division of labor, to maintenance personnel according to the category of the medical equipment consisted of various professional groups. If under the condition of insufficient maintenance engineers, the division of labor can be carried out according to the professional division of labor and the division of the disease area; Appropriate configuration must be inspection tools to improve the daily maintenance work, combined with the actual work situation, inventory varieties and amount of damageable parts, in order to improve the speed of maintenance, maintenance personnel in the maintenance after the completion of detailed records, fill in equipment maintenance and 
record the spare parts, maintenance time, site conditions, maintenance; Input devices within the network, can test at any time and query, observe the usage of partial equipment, make the equipment instrument management more scientific, standardized and institutionalized, improve equipment utilization.

\subsection{Continuously improve the quality of maintenance engineers.}

Medical engineering technique is a very professional job, to do a good job in modern medical equipment maintenance work, must have a broad solid theoretical foundation for knowledge and good foreign language level, familiar with the theory of all kinds of engineering knowledge and mastering business skills, has the good professional spirit and ethical values.

\section{How to develop our own biomedical engineering and medical equipment?}

Biomedical engineering and medical equipment between the two complement each other, on the one hand, biomedical engineering is a foundation, it will create a new topic of study medical equipment, at the same time, demand for clinical medical equipment information will cause a new research direction. We have very much, strong ability of scientific research personnel, research topic did very well, but the results into the practical application of the scale is not large, medical equipment manufacturing enterprises are low imitation foreign existing products or product technology content is low, simple, the craft is poor. How to develop our own biomedical engineering and medical equipment equipment need to think, reference:

(1) Strengthen communication between scientific researchers and production enterprises;

(2) Strengthen the biomedical engineering of scientific researchers, clinicians and medical units.

Communication between service personnel; accelerate the transformation of technology to practical application. Now more favourable environment system, the innovation and achievements transformation of science and technology has become our basic development direction, believe that as long as you work hard, biomedical engineering and medical equipment development prospects look good.

\section{References}

[1] Johnson D H, Bidez M W, Delucas L J. Hazard Analysis and Risk Assessment in the Development of Biomedical Drug Formulation Equipment [J]. Annals of Biomedical Engineering, 2012, 40(4):898-906.

[2] Rubin J B, Paltiel A D, Saltzman W M. Are We Studying What Matters? Health Priorities and NIH-Funded Biomedical Engineering Research [J]. Annals of Biomedical Engineering, 2010, 38(7):2237-2251.

[3] Stukel J M, Goss M, Zhou H, et al. Development of a High-Throughput Ultrasound Technique for the Analysis of Tissue Engineering Constructs [J]. Annals of Biomedical Engineering, 2016, 44(3):793.

[4] Lee S, Chang W L, Kim C S. FEA Study on the Stress Distributions in the Polymer Coatings of Cardiovascular Drug-Eluting Stent Medical Devices [J]. Annals of Biomedical Engineering, 2014, 42(9):1952-1965.

[5] Mohammadi S, Postnikoff C, Wright A M, et al. Design and Development of an In Vitro, Tear Replenishment System [J]. Annals of Biomedical Engineering, 2014, 42(9):1923-1931. 